\title{
Mathematical Modelling for Joining Boron Nitride Graphene with Other BN Nanostructures
}

\author{
Nawa A. Alshammari \\ Department of Mathematics, College of Science and Theoretical Studies, Saudi Electronic University, Saudi Arabia \\ Correspondence should be addressed to Nawa A. Alshammari; n.alshammari@seu.edu.sa
}

Received 19 April 2020; Revised 11 August 2020; Accepted 12 August 2020; Published 30 August 2020

Academic Editor: Ruben Specogna

Copyright (c) 2020 Nawa A. Alshammari. This is an open access article distributed under the Creative Commons Attribution License, which permits unrestricted use, distribution, and reproduction in any medium, provided the original work is properly cited.

\begin{abstract}
Boron nitride $(\mathrm{BN})$ nanomaterials such as boron nitride graphenes, boron nitride nanotubes, and boron nitride nanocones are attracting attention among the most promising nanomaterials due to their physical, chemical, and electronic properties when compared to other nanomaterials. BN nanomaterials suggest many exciting potential applications in various fields. Joining between BN nanostructures gives new enhanced structures with outstanding properties and potential applications for design of probes for scanning tunnelling microscopy and other nanoscale devices. This paper uses calculus of variations to model the joining between BN graphene with other BN nanostructures: BNNTs and BNNCs. Furthermore, during the joining between these $\mathrm{BN}$ nanostructures, this research examines two models which are depending on the curvature of the join profile. For the first case, Model I refers to when the join profile only includes positive curvature where for the second case, Model II is considered for both positive and negative curvatures. Thus, the purpose of this research is to formulate the basic underlying structure to present simple models based on joining BN graphene to other BN nanostructures.
\end{abstract}

\section{Introduction}

Materials in nanoscale have attracted research in different scientific fields because of their chemical, physical, and electronic properties. In particular, carbon in nanoscale has different shapes and structures such as carbon nanotubes, carbon nanocones, carbon nanosheets (graphene), and fullerene.

Due to similarity to carbon nanostructures, boron nitride nanostructures have received specific attention due to their remarkable properties that lead them to a wide range of applications such as developing many nanoscale devices $[1,2]$. Hexagonal BN structure involves boron and nitrogen atoms bound by strong covalent bonds. Moreover, many forms of boron nitride in nanoscale have been reported in the previous literature such as boron nitride nanotubes (BNNTs), boron nitride graphene (BN graphene), boron nitride nanocones (BNNCs), and boron nitride fullerene (BN fullerene) [3].

BNNTs were first discovered in 1994 [4] and contain a tubular structure as carbon nanotubes with replacing carbon atoms by boron and nitrogen atoms arranging in a hexagonal lattice. BNNTs have gained significant attention in recent years because of their interesting properties that are not available in other nanomaterials. While they are structurally close to carbon nanotubes, BNNTs have completely different physical properties. For example, BNNTs have mechanical, thermal, electrical, and chemical properties like high tensile stiffness and high thermal conductivity. Also, their electronic properties are independent of their chirality and radii of the tube [4-6].

Another boron nitride nanostructure has attracted much attention, that is, boron nitride graphene. Geometrically, it is similar to carbon nanosheet with boron and nitrogen atoms instead of carbon atoms. BN graphene has physical and chemical distinct properties, such as high-temperature stability, intrinsic electrical insulation, and semiconducting and antioxidation ability. As a result, BN graphene has a promising application in various areas; namely, it is used as a two-dimensional filler, nonwetting coatings, and field emitters [7-9].

Boron nitride nanocones (BNNCs) are important structures of BN nanostructures. These conical structures have 
been considered in many studies experimentally and theoretically. Their special structure offers suitability for fabrication of nanodevices such as electron field emitters, sensors, nanoindenters, and probe tips. Carbon nanocones (CNCs) can be formed by curling the fan-shaped graphite sheet with a disclination angle of an integer multiple of $60^{\circ}$ where BNNCs can be produced by only rolling up the white graphene with a disclination angle of an integer multiple of $120^{\circ}$ [10]. In addition, carbon nanocones have five possible structures depending on the number of pentagons, and the conical structure of boron nitride has been studied using transmission electron microscopy and observed their angles to be $84^{\circ}, 19.2^{\circ}$, and $38.9^{\circ}$ [11].

New structures produced from the joining between two nanostructures can enhance the physiochemical and electrochemical performances of the joined materials. For example, carbon nanotubes and carbon nanosheets are useful in nanosensors and nanooscillators, and connections of these carbon nanostructures may also be employed in the same applications. Furthermore, the same applications exist for joining BNNTs and BN graphene [1]. Moreover, the composite structures are useful for the design of probes for scanning tunnelling microscopy, energy storage, and other electronic devices as carriers for drug delivery [2].

Research in [12] minimizes the elastic energy (which depends on the axial curvature only) using calculus of variations to determine the joining area between two carbon nanostructures. The chemical issues are ignored in this model such as the positions of the carbon atoms and bonds. Based on the length constraint of the joining between two nanostructures, the curvature of the join curve can be either positive or both positive and negative. In addition, the study in [13] studies different cases for joining between carbon nanostructures using the same method described above, including the joining of two carbon nanotubes, tube with fullerene, tube with cone, and two fullerenes. Furthermore, this model has been extended to study the joining of some boron nitride nanostructures as in [14].

In this paper, we use the same model to determine the shape of the joining between BN graphene with BNNTs and BNNCs, where the arc length of the curve and the size of the defect in the $\mathrm{BN}$ graphene are specified, and the distance of the BNNTs and BNNCs from the BN graphene is not prescribed and is determined as a part of the solution.

Finally, we comment that using Willmore energy which depends on the axial and rotational curvatures to determine the joining between two nanostructures gives rise to similar join profiles of using elastic energy as studied in [15]. Moreover, the mathematical techniques in [16-19] have similar ideas as in this research.

In the following section, we state the fundamental equations of the calculus of variations to model the joining region between BN graphene with BNNTs and BNNCs. In Subsection 2.1, the curvature is assumed to remain positive in the join region, and we denote this case as Model I. Subsection 2.2 presents Model II which is assumed to have two join regions, one of positive curvature and the second of negative curvature. Results and discussion are given in Section 4. Section 4 provides the summary.

\section{Model}

Here, the basic variational equations of the model that is used to join boron nitride graphene to other boron nitride nanostructures are formulated. In details, variational calculus is used to identify the curve adopted by a line smoothly connecting a $\mathrm{BN}$ graphene base to a vertical other boron nitride nanostructure, where the arc length of the joining curve and the defect site at the BN graphene base are specified. Therefore, the distance in the $y$-direction $y_{0}$ of the joining to the tube or cone is not specified, and it is found as a part of the solution.

We position the first nanostructure (BN graphene) in the $(x, z)$-plane with a circular defect of radius $x_{0}$ centred on the origin. Assuming that a second nanostructure (BNNTs or BNNCs) of radius $a$ is located with its axis colinear with the $y$-axis starting from an unknown positive distance above the $(x, z)$-plane that is denoted by $y_{0}$, the defect and second nanostructure are assumed to be rotationally symmetric about the $y$-axis; the problem can be reduced to two-dimensional $(x, y)$-plane. The total prescribed arc length $\ell$ is assumed to connect the defects at the points $\left(x_{0}, 0\right)$ and $\left(a, y_{0}\right)$. We comment that this technique is used first by Cox and Hill in [12] and then by Baowan et al. [2, 13].

Using calculus of variations to find the curve $y(x)$, with an element of arc length $d s$, minimizes the energy functional $J[y]$ that is given by

$$
J[y]=\int_{0}^{\ell} \kappa^{2} d s+\lambda \int_{0}^{\ell} d s
$$

where $\kappa$ is the curvature, $\lambda$ is a Lagrange multiplier corresponding to the fixed length constraint, and $l$ is the length of the join curve. The boundaries of the join region are $x_{0}$ and $x_{1}$, where at $x=x_{0}$, we have $s=0$, and at $x=x_{1}$, we have $s=\ell$. The curve in two dimensions described as a graph $y=y(x)$, we have $\kappa=\ddot{y} /\left(1+\dot{y}^{2}\right)^{3 / 2}$, and $d s=\left(1+\dot{y}^{2}\right)^{1 / 2} d x$, then equation (1) becomes

$$
J[y]=\int_{a}^{x_{0}} \frac{\ddot{y}^{2}}{\left(1+\dot{y}^{2}\right)^{5 / 2}} d x+\lambda \int_{a}^{x_{0}}\left(1+\dot{y}^{2}\right)^{1 / 2} d x
$$

where in this paper, dot denotes differentiation with respect to $x$. We have two different models based on the curvature's sign, and the join curvature remains positive as shown in Figure 1 and both positive and negative curvatures as in Figure 2. For these models, we impose the continuity boundary conditions at the first nanostructure ( $\mathrm{BN}$ graphene) where $\theta_{0}=0$,

$$
\begin{aligned}
& y\left(x_{0}\right)=0, \\
& \dot{y}\left(x_{0}\right)=0 .
\end{aligned}
$$




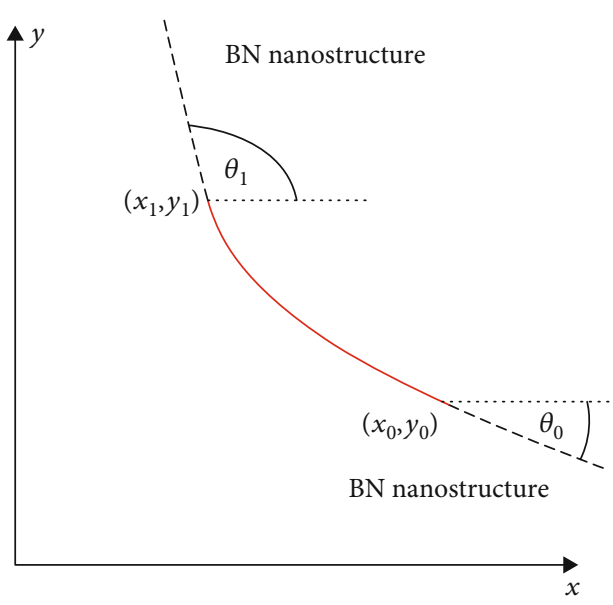

Figure 1: Model I: positive curvature [2].

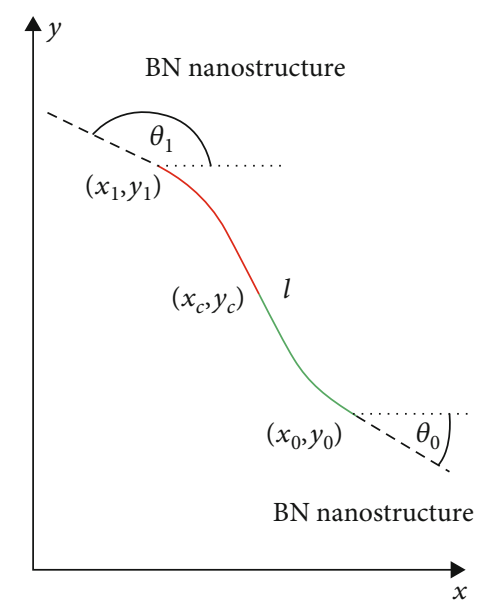

FIgURE 2: Model II: positive and negative curvatures [2].

To determine the boundary conditions at the second nanostructure (BNNTs or BNNCs), we integrate by parts to derive the standard equation

$$
\begin{aligned}
\delta J[y]= & {\left[\left(F_{\dot{y}}-\frac{d}{d x} F_{\ddot{y}}\right) \delta y+F_{\ddot{y}} \delta \dot{y}\right]_{x_{0}}^{x_{1}} } \\
& +\int_{x_{0}}^{x_{1}}\left(F_{y}-\frac{d}{d x} F_{\dot{y}}+\frac{d^{2}}{d x^{2}} F_{\ddot{y}}\right) \delta y d x,
\end{aligned}
$$

where subscripts denote partial derivatives, and here, the function $F$ is given by

$$
F(\dot{y}, \ddot{y})=\frac{\ddot{y}^{2}}{\left(1+\dot{y}^{2}\right)^{5 / 2}}+\lambda\left(1+\dot{y}^{2}\right)^{1 / 2} .
$$

In this case, only $\dot{y}$ is prescribed at $x=a$, as $y_{0}$ is unknown, and at $x=a$, we require the natural or alternative boundary condition given by

$$
\left[\left(F_{\dot{y}}-\frac{d}{d x} F_{\ddot{y}}\right)\right]_{x=a}=0 .
$$

For the case of BNNTs, $\theta_{1}=\pi / 2$. The value of $\dot{y}$ in Model I ranges from 0 at $x=x_{0}$ to $-\infty$ at $x=a$. Thus, the boundary condition for this model is $\dot{y}(a)=-\infty$. For Model II, $\dot{y}$ ranges from 0 to $-\infty$, where it changes sign and then ranges from $\infty$ down to some finite positive value before turning to $\infty$. Therefore, the boundary condition in the case of Model II is $\dot{y}(a)=\infty$. For both models, we have at $x=a$, equation (6) along with the boundary condition for Model I:

$$
\dot{y}(a)=-\infty
$$

and for Model II is

$$
\dot{y}(a)=\infty \text {. }
$$

Noting that, for the case of BNNCs, $\theta_{1}$ is the angle of the nanocone. From equation (4), we have the usual EulerLagrange equation for $F(x, y, \dot{y}, \ddot{y})$, which is given by

$$
F_{y}-\frac{d}{d x} F_{\dot{y}}+\frac{d^{2}}{d x^{2}} F_{\ddot{y}}=0
$$

However, since our function $f(\dot{y}, \ddot{y})$ is independent of $y$, integrating equation (9) gives

$$
F_{\dot{y}}-\frac{d}{d x} F_{\ddot{y}}=C_{1} \text {, }
$$

where $C_{1}$ is an arbitrary constant. From the natural boundary condition in equation (6), we find that $C_{1}=0$, so we obtain

$$
F_{\dot{y}}=\frac{d}{d x} F_{\ddot{y}}
$$

Using the definition of the full derivative, we have

$$
\frac{d F}{d x}=F_{x}+\dot{y} F_{y}+\ddot{y} F_{\dot{y}}+\dddot{y} F_{\ddot{y}} .
$$

Since $F_{x}=F_{y}=0$, from equation (11), we obtain

$$
\frac{d}{d x}\left(F-\ddot{y} F_{\ddot{y}}\right)=0,
$$

by integrating with respect to $x$, we get

$$
F-\ddot{y} F_{\ddot{y}}=-\alpha,
$$

where $\alpha$ is an arbitrary constant. Now, we substitute equation (5) into equation (14) and obtain

$$
\frac{\ddot{y}^{2}}{\left(1+\dot{y}^{2}\right)^{3}}=\lambda+\frac{\alpha}{\left(1+\dot{y}^{2}\right)^{1 / 2}} .
$$


As a result, we can write the curvature $\kappa$ as

$$
\kappa= \pm\left(\lambda+\frac{\alpha}{\left(1+\dot{y}^{2}\right)^{1 / 2}}\right)^{1 / 2}
$$

2.1. Model I: Positive Curvature. The curvature in Model I is positive through the arc length $l$ as shown in Figures 3 and 4 . As a result, we only consider the positive case from equation (16). Using $\dot{y}=\tan \theta$, equation (8) becomes

$$
\kappa=(\lambda+\alpha \cos \theta)^{1 / 2} .
$$

If $\kappa=\ddot{y} /\left(1+\dot{y}^{2}\right)^{3 / 2}$, and using the same substitution for $\dot{y}$, we obtain

$$
\frac{d y}{d \theta}=\frac{\sin \theta}{(\lambda+\alpha \cos \theta)^{1 / 2}} .
$$

By introducing the constant $k=[(\lambda+\alpha) / 2 \alpha]^{1 / 2}$, and a new parametric variable $\phi$ which is defined by

$$
\cos \theta=1-2 k^{2} \sin ^{2} \phi
$$

then, equation (18) can be written as

$$
\frac{d \theta}{d \phi}=\frac{2 k \cos \theta}{\left(1-k^{2} \sin ^{2} \phi\right)^{1 / 2}},
$$

and on introducing another constant $\beta=(2 / \alpha)^{1 / 2}$, we obtain

$$
\frac{d y}{d \phi}=2 \beta k \sin \phi
$$

By the integration and the boundary condition at the point $\left(x_{0}, y_{0}\right)$, we obtain

$$
y(\phi)=2 \beta k(1-\cos \phi) .
$$

We note that equation (22) can be considered as a parametric equation for $y$ in terms of the parameter $\phi$ and denote the value of $\phi$ at the point where $\theta=-\pi / 2$, with $\phi_{0}=\sin ^{-1}(1 / \sqrt{2} k)$, and for Model I, $-\phi_{0}<\phi \leq 0$.

Similarly, we derive

$$
\frac{d x}{d \theta}=\frac{\cos \theta}{(\lambda+\alpha \cos \theta)^{1 / 2}},
$$

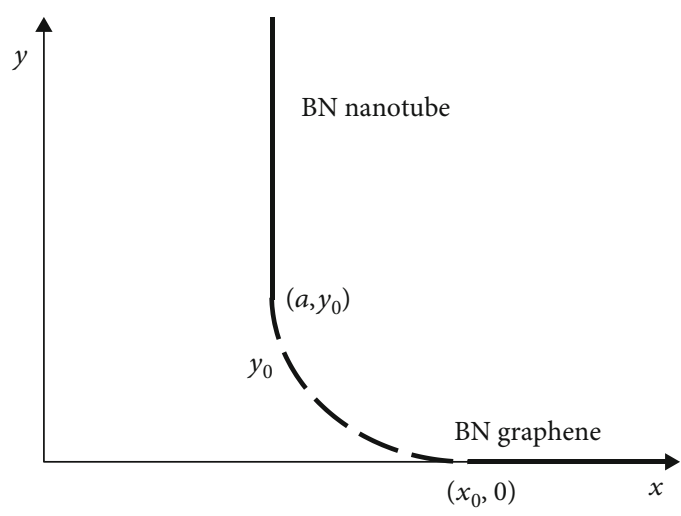

FIgURE 3: Joining of BN graphene and $\mathrm{BN}$ nanotube.

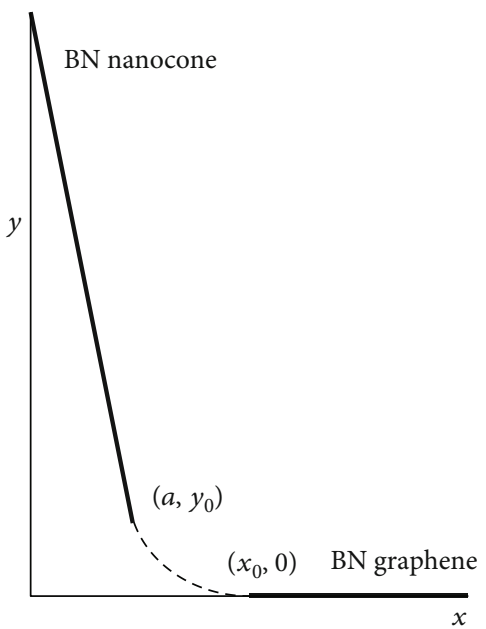

FIGURE 4: Joining of BN graphene and BN nanocone.

and in terms of the variable $\phi$, we have

$$
\begin{aligned}
\frac{d x}{d \phi} & =\beta \frac{\left(1-2 k^{2} \sin ^{2} \phi\right)}{\left(1-k^{2} \sin ^{2} \phi\right)^{1 / 2}} \\
& =\beta\left[2\left(1-k^{2} \sin ^{2} \phi\right)^{1 / 2}-\left(1-k^{2} \sin ^{2} \phi\right)^{-1 / 2}\right] .
\end{aligned}
$$

Integrating (24) gives

$$
x(\phi)=x_{0}+\beta([2 E(\phi, k)-F(\phi, k)]
$$

where $F(\phi, k)$ and $E(\phi, k)$ denote the usual Legendre incomplete elliptic integrals of the first and second kinds, respectively. Using equations (22) and (25), we obtain

$$
\begin{aligned}
x_{0}-a & =\beta\left(\left[2 E\left(\phi_{0}, k\right)-F\left(\phi_{0}, k\right)\right],\right. \\
y_{0} & =2 \beta k\left(1-\cos \phi_{0}\right) .
\end{aligned}
$$


From the definition of the arc length, we have

$$
\ell=\int_{a}^{x_{0}}\left(1+\dot{y}^{2}\right)^{1 / 2} d x
$$

Upon substituting $\dot{y}=\tan \theta$, changing the parameter to $\phi$ as in $\cos \theta=1-2 k^{2} \sin ^{2} \phi$ and integrating, we have

$$
\ell=\beta F\left(\phi_{0}, k\right)
$$

Now, we define a dimensionless parameter $\mu=\left(x_{0}-a\right) / \ell$, which can be shown to be

$$
\mu=2\left(\frac{E\left(\phi_{0}, k\right)}{F\left(\phi_{0}, k\right)}\right)-1
$$

where $\mu=\left(x_{0}-a\right) / \ell$ and $-1<\mu<1$. For prescribed values of $a, x_{0}$, and $\ell$, equation (29) can be solved numerically to determine the value of $k$. Then, substituting $k$ into equation (28), the value of $\beta$ can be determined, and therefore, $y_{0}$ can be obtained from equation (28).

2.2. Model II: Positive and Negative Curvatures. For the first region, the curvature is positive at $\left(x_{0}, 0\right)$ up until the point $\left(x_{c}, y_{c}\right)$, where the curvature changes sign to be negative until the point $\left(a, y_{0}\right)$ is reached as in Figures 5 and 6. At $\left(x_{c}, y_{c}\right), \kappa=0$, and by solving equation (17), we have $\theta_{c}=-\cos ^{-1}(-\gamma / \alpha)$, noting that from geometrical considerations, we have $-\pi<\theta_{c}<-\phi / 2$. By making the substitution used in equation (19) for $\phi$, we have $\phi_{c}=-\pi / 2$. By substituting $\phi_{c}$ into equations (22) and (25), we can calculate $x_{c}$ and $y_{c}$,

$$
\begin{aligned}
& x_{c}=x_{0}-\beta\{2[E(k)-K(k)]\}, \\
& y_{c}=2 \beta k,
\end{aligned}
$$

noting that $K(k)$ and $E(k)$ are the complete elliptic integrals of the first and second kinds, respectively. In the second joining region which is between $\left(x_{c}, y_{c}\right)$ and $\left(a, y_{0}\right)$, we take the negative sign of equation (16), and following the same method used in Model I, we obtain

$$
x(\phi)=x_{0}-\beta\{2[2 E(k)-E(-\phi, k)]-[2 K(k)-F(-\phi, k)]\},
$$

$$
y(\phi)=2 \beta k(1+\cos \phi) .
$$

As a result, from the boundary conditions at the point $\left(a, y_{0}\right)$, we know that $\phi=-\phi_{0}$. By substitution into (32), we find

$$
\begin{aligned}
x_{0}-a & =\beta\left\{2\left[2 E(k)-E\left(\phi_{0}, k\right)\right]-\left[2 K(k)-F\left(\phi_{0}, k\right)\right]\right\}, \\
y_{0} & =2 \beta k\left(1+\cos \phi_{0}\right) .
\end{aligned}
$$

In this model, the arc length is determined in two parts, which is given by

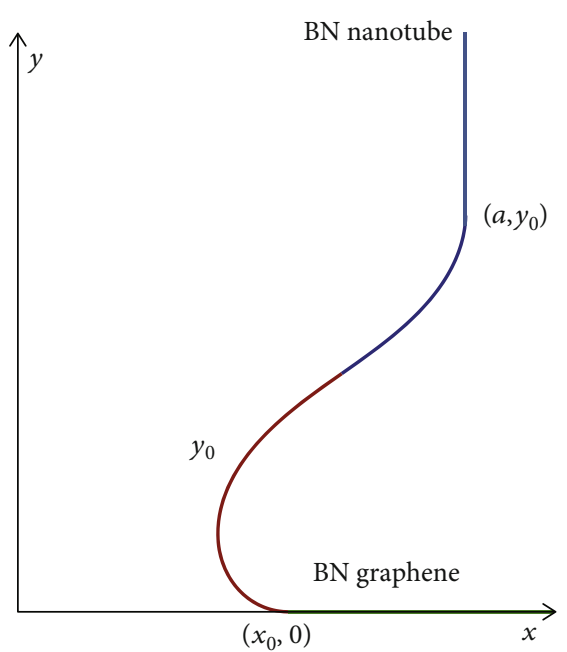

FIGURE 5: Joining of BN graphene and BN nanotube.

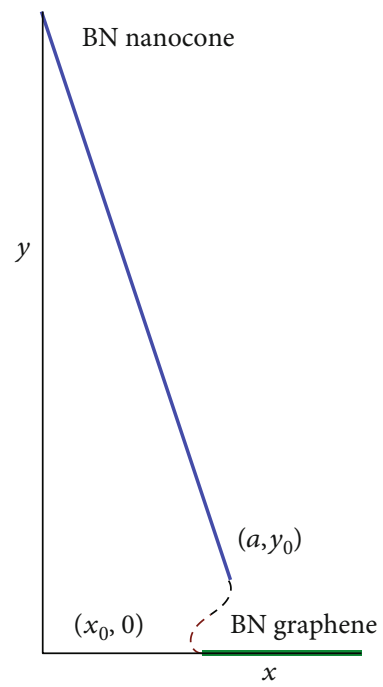

Figure 6: Joining of $\mathrm{BN}$ graphene and $\mathrm{BN}$ nanocone.

$\ell=\int_{\theta_{c}}^{0}\left(1+\dot{y}^{2}\right)^{1 / 2} d x+\int_{\theta_{c}}^{-\phi / 2}\left(1+\dot{y}^{2}\right)^{1 / 2} d x=\beta\left[2 K(k)-F\left(\phi_{0}, k\right)\right]$

As a result, we find a dimensionless parameter $\mu=$ $\left(x_{0}-a\right) / \ell$ for this case to be

$$
\mu=2\left(\frac{2 E(k)-E\left(\phi_{0}, k\right)}{2 K(k)-F\left(\phi_{0}, k\right)}\right)-1
$$

Also, for prescribed values of $x_{0}, x_{1}$, and $\ell$, we can solve equation (36) numerically to find the value of $k$, and by substituting $k$ into equation (35), we can determine the value of $\beta$ so that $y_{0}$ can be determined from equation (34) [12]. Here, we note that equation (29) coincides with (36) for the value $k=1 / \sqrt{2}$, and the value of $\mu$ at this point is 


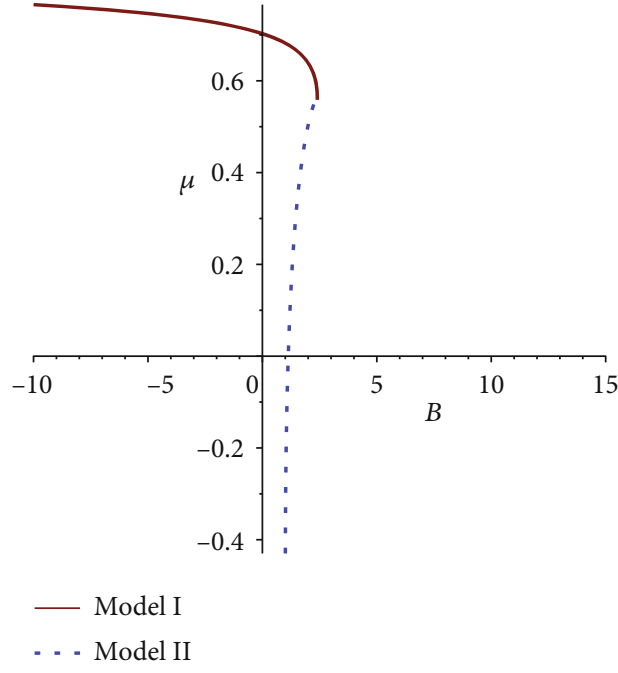

Figure 7: The relation between the characteristic parameter $\mu$ and constant $B$ for Model I and Model II [2].

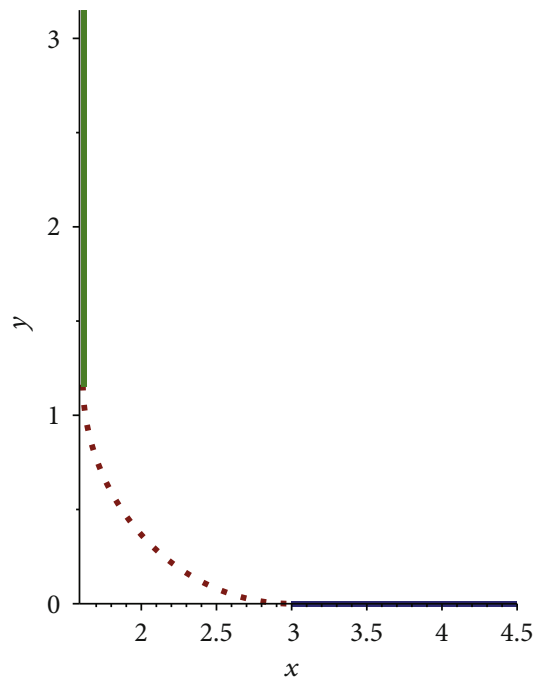

Figure 8: Plot of the join curve $y=y(x)$ of Model I for joining BN nanotube with $\mathrm{BN}$ graphene where $B=-4$ and $\ell=2$.

$$
\mu_{0}=2\left(\frac{E(1 / \sqrt{2})}{K(1 / \sqrt{2})}\right)-1=0.4569465810 \cdots
$$

\section{Results}

In this section, we investigate the numerical solutions for equations (29) and (37) when they are characterized by the nondimensional parameter $\mu=\left(x_{0}-a\right) / \ell$, subjecting to the constraint $-1<\mu<1$, as shown in [12]. Figure 7 shows that the three distinct regions are evident: the first one is where the parameter $B$ is negative that is corresponding to Model I; the second region is $0<B \leq 2$, which also corresponds to Model I; and the third region is $1<B \leq 2$, which corresponds to Model II.

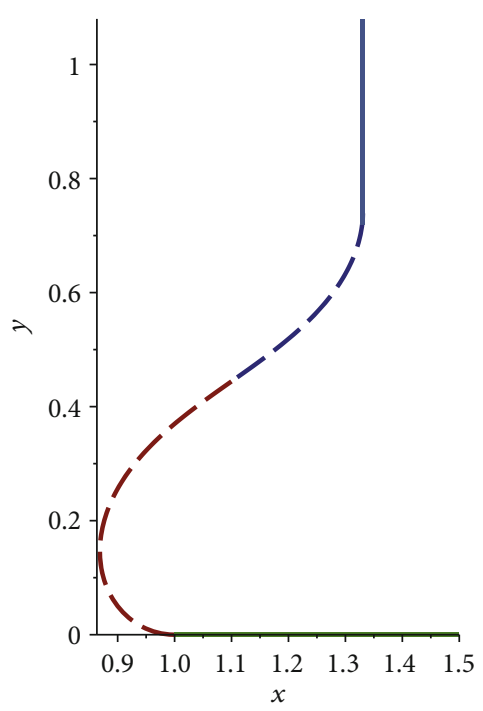

FIgURE 9: Plot of the join curve $y=y(x)$ of Model II for joining BN nanotube with $\mathrm{BN}$ graphene where $B=1.1$ and $\ell=1$.

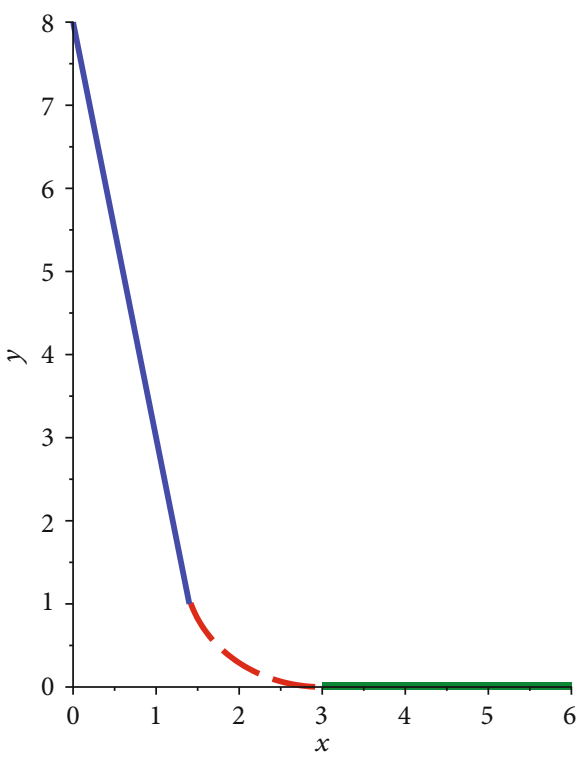

FIgURe 10: Plot of the join curve $y=y(x)$ of Model I for joining BN nanocone $38.9^{\circ}$ with $\mathrm{BN}$ graphene where $B=-4$ and $\ell=2$.

Results for the joining between $\mathrm{BN}$ graphene and $\mathrm{BN}$ nanotube for both models I and II are shown in Figures 8 and 9, respectively. Furthermore, Figures 10 and 11 illustrate the join profile for $\mathrm{BN}$ graphene and $\mathrm{BN}$ nanocone with both models.

\section{Conclusion}

In this paper, conventional applied mathematical modelling is considered to identify an approximate analytical solution to the shape of a surface joining $\mathrm{BN}$ graphene to other $\mathrm{BN}$ nanostructures. The resulted improved nanostructures could be useful for the design of probes for scanning tunnelling microscopy and other nanoscale devices. Using calculus of variations, this research minimizes the elastic energy of the 


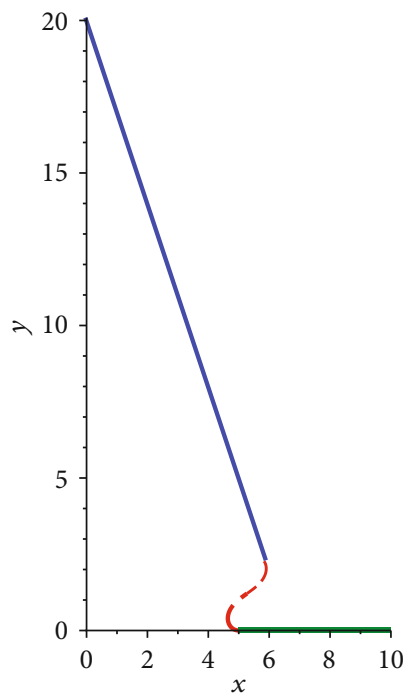

Figure 11: Plot of the join curve $y=y(x)$ of Model II for joining BN nanocone $38.9^{\circ}$ with graphene sheet where $B=1.1$ and $\ell=1$.

joining curve which relates to minimization of the covalent bond energy. In particular, the joining is considered based on two different models that depend on the sign of the curvature: Model I refers to positive curvature only and Model II refers to positive and negative curvatures. The main purpose of this work is to formulate the axially symmetric model to have a reference basis for the comparison of real physical structures. As a result, these models lead to significative approximations to complex structures that are formulated by these certain $\mathrm{BN}$ nanostructures, $\mathrm{BN}$ graphene with two different $\mathrm{BN}$ nanostructures, BNNTs and BNNCs. Finally, other different $\mathrm{BN}$ nanostructures can be joined using the same model, and this is an area for future work.

\section{Data Availability}

No data were used to support this study.

\section{Conflicts of Interest}

The author declares no competing interests.

\section{Authors' Contributions}

N.A. wrote the main manuscript text, prepared all figures, and reviewed the manuscript.

\section{References}

[1] S. Rouhi, R. Ansari, and A. Shahnazari, "Vibrational characteristics of single-layered boron nitride nanosheet/single-walled boron nitride nanotube junctions using finite element modeling," Materials Research Express, vol. 3, no. 12, article 125027, 2016.

[2] N. A. Alshammari, N. Thamwattana, J. McCoy, B. Duangkamon, B. Cox, and J. Hill, "Modelling joining of various carbon nanostructures using calculus of variations," Dynamics of Continuous, Discrete and Impulsive Systems Series B: Applications and Algorithms, vol. 25, pp. 307-339, 2018.
[3] S. Ramon, Boron nitride and carbon nanostructures: synthesis, characterization and $a b$ initio calculations, [Ph.D. thesis], University of San Luis Potosi, 2015.

[4] A. Rubio, J. L. Corkill, and M. L. Cohen, "Theory of graphitic boron nitride nanotubes," Physical Review B, vol. 49, no. 7, pp. 5081-5084, 1994.

[5] J. H. Kim, T. V. Pham, J. H. Hwang, C. S. Kim, and M. J. Kim, "Boron nitride nanotubes: synthesis and applications," Nano Convergence, vol. 5, no. 1, p. 17, 2018.

[6] C. Lee, S. Bhandari, B. Tiwari, N. Yapici, D. Zhang, and Y. Yap, "Boron nitride nanotubes: recent advances in their synthesis, functionalization, and applications," Molecules, vol. 21, no. 7, p. 922, 2016.

[7] M. Du, X. Li, A. Wang, Y. Wu, X. Hao, and M. Zhao, "Onestep exfoliation and fluorination of boron nitride nanosheets and a study of their magnetic properties," Angewandte Chemie International Edition, vol. 53, no. 14, pp. 3645-3649, 2014.

[8] J. Zhou, Q. Wang, Q. Sun, and P. Jena, "Electronic and magnetic properties of a $\mathrm{BN}$ sheet decorated with hydrogen and fluorine," Physical Review B, vol. 81, no. 8, 2010.

[9] B. J. Cox, D. Baowan, W. Bacsa, and J. M. Hill, "Relating elasticity and graphene folding conformation," RSC Advances, vol. 5, no. 71, pp. 57515-57520, 2015.

[10] J. W. Yan and K. M. Liew, "Predicting elastic properties of single-walled boron nitride nanotubes and nanocones using an atomistic-continuum approach," Composite Structures, vol. 125, pp. 489-498, 2015.

[11] B. Duangkamon, Mathematical modelling of nanostructures, [Ph.D. thesis], University of Wollongong, 2008.

[12] B. J. Cox and J. M. Hill, "A variational approach to the perpendicular joining of nanotubes to plane sheets," Journal of Physics A: Mathematical and Theoretical, vol. 41, no. 12, article 125203, 2008.

[13] D. Baowan, B. J. Cox, and J. M. Hill, "Determination of join regions between carbon nanostructures using variational calculus," The ANZIAM Journal, vol. 54, no. 4, pp. 221-247, 2013.

[14] N. Alshammari, "Joining between boron nitride nanocones and nanotubes," Advances in Mathematical Physics, vol. 2020, Article ID 5631684, 6 pages, 2020.

[15] P. Sripaturad, N. A. Alshammari, N. Thamwattana, J. A. McCoy, and D. Baowan, "Willmore energy for joining of carbon nanostructures," Philosophical Magazine, vol. 98, no. 16, pp. 1511-1524, 2018.

[16] L. Zhang, M. B. Arain, M. M. Bhatti, A. Zeeshan, and H. HalSulami, "Effects of magnetic reynolds number on swimming of gyrotactic microorganisms between rotating circular plates filled with nanofluids," Applied Mathematics and Mechanics, vol. 41, no. 4, pp. 637-654, 2020.

[17] M. Marin and S. Nicaise, "Existence and stability results for thermoelastic dipolar bodies with double porosity," Continuum Mechanics and Thermodynamics, vol. 28, no. 6, pp. 1645-1657, 2016.

[18] M. Marin, R. Ellahi, and A. Chirila, "On solutions of SaintVenant's problem for elastic dipolar bodies with voids," Carpathian Journal of Mathematics, vol. 33, no. 2, pp. 219-232, 2017.

[19] L. Barbu and A. E. Nicolescu, "An overdetermined problem for a class of anisotropic equations in a cylindrical domain," Mathematical Methods in the Applied Sciences, vol. 43, no. 9, pp. 6117-6125, 2020. 